\title{
DRAFT: EXPERIMENTAL DETERMINATION OF DOMINANT MULTIPLIERS IN MILLING PROCESS BY MEANS OF HOMOGENEOUS COORDINATE TRANSFORMATION
}

\author{
Adam K. Kiss \\ Department of Applied Mechanics \\ Budapest University of \\ Technology and Economics \\ Budapest, Hungary \\ Email: kiss_a@mm.bme.hu
}

\author{
Daniel Bachrathy \\ Department of Applied Mechanics \\ Budapest University of \\ Technology and Economics \\ Budapest, Hungary \\ Email: bachrathy@mm.bme.hu
}

\author{
Gabor Stepan \\ Department of Applied Mechanics \\ Budapest University of \\ Technology and Economics \\ Budapest, Hungary \\ Email: stepan@mm.bme.hu
}

\begin{abstract}
In this contribution, a chatter detection method is investigated for milling operations. The proposed approach can give not only qualitative condition (stable or unstable), but a quantitative measure of stability. For this purpose, it requires an external excitation of stable machining condition. Transient vibration of the perturbation is captured by means of stroboscopic section, and the corresponding monodromy operator is approximated by its projection to the subspace of the dominant modes. The monodromy matrix is determined with the application of homogeneous coordinate representation. Then, the periodic solution and the dominant characteristic multipliers are calculated and their modulus determines the quantitative measure of stability condition.
\end{abstract}

\section{INTRODUCTION}

In the production and manufacturing industry, milling is a widely used method. However, high material removal rate often cannot be established in practice due to the instability of the cutting process. These vibrations are classified into two groups [1]. One of them is the self-excited vibration which evolves in case of stability loss related to the surface regeneration effect [2]. This effect is usually modelled with delay-differential equations (DDE) [3]. The other type of vibration is the forced periodic vibration [4]. The so-called stability chart [5] presents the chatter- free (stable) domains of technological parameters which are usually illustrated in the plane of the spindle speed and the axial immersion. It can be calculated by frequency domain $[6,7]$ or time-domain methods [8-10].

The experimental construction of these maps usually based on chatter detection techniques [11]. Some of them investigate the resultant spectrum of the sensors (typically industrial microphone and/or accelerometer) in frequency domain [12-14]. Other technique analyse the sampled signals in time domain [15-17], or examine the quality of the milled surface [18]. These techniques usually give qualitative condition to the stability of milling process, whether it is stable or not. Near to the stability boundaries, measurement points are sometimes marked as marginal. Therefore, the comparison is difficult between the theoretically calculated stability chart and the reconstructed diagram.

In this paper, we introduce a method which is capable to provide a quantitative measure of stability, which can be directly compared to the theoretically predicted stability condition. For this purpose, a good choice is to compare the dominant characteristic multipliers of the related Floquet theory of DDEs [8]. To obtain them from experiments, it requires additional excitation during stable machining condition, and the resultant transient vibration has to be captured. Then, the dominant multipliers can be approximated in least-square sense [15]. In addition, the modulus of the dominant multiplier can be inferred to the damping of 


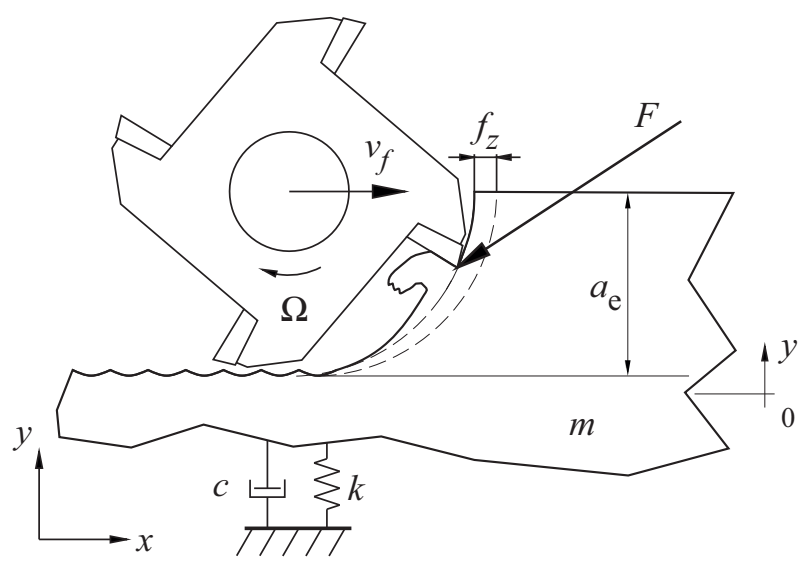

FIGURE 1. MECHANICAL MODEL OF THE MILLING PROCESS FOR $N$ NUMBER OF FLUTED TOOL. PARAMETERS: $\Omega$ SPINDLE SPEED

the perturbed solution. With this process, the change of stability condition that is the modulus of multipliers can be traced without switching between stable and unstable conditions. The stability boundary can be determined precisely by means of interpolation between measurement points, furthermore, extrapolation can be used to predict the distance from the stability boundary based on stable measurements points, only.

The accuracy of the proposed method is investigated based on a widely-used setup, for which the corresponding mechanical model of the milling process is well-developed. Therefore, a Single-Degree-of-Freedom (SDoF) flexure is used in the presented method, which can be found in [19].

\section{MECHANICAL MODEL OF MILLING}

In this section, the mechanical model of the milling process is described. The dynamical model of the test rig is presented in Fig 1 [19], where the milling tool is assumed to be rigid and the workpiece is flexible along the $y$ direction. One-Degree-ofFreedom regenerative milling model is used with the corresponding governing equation $[1,2,8,20]$

$$
m \ddot{y}(t)+c \dot{y}(t)+k y(t)=F_{y}(y(t), y(t-\tau)),
$$

where $m, c$ and $k$ are the mass, damping and stiffness parameters, respectively. The general coordinate is denoted by $y(t)$, and the cutting force acting on the workpiece is $F_{y}(y(t), y(t-\tau))$. The resultant force is linearly proportional to the area of the chip cross section which has time periodic component due to the intermittent milling process, and it is influenced by the actual $y(t)$ and the previous position $y(t-\tau)$ due to the regenerative effect [2]. The time delay $\tau$ and the time period of the system $T=2 \pi /(\Omega N)$ are equivalent for the considered straight fluted tool.
According to [8], the general solution $y(t)$ of Eq. (1) can be written as a small perturbation $\eta(t)$ around its periodic term $y_{\mathrm{p}}(t)$ as

$$
y(t)=y_{\mathrm{p}}(t)+\eta(t)
$$

where $y_{\mathrm{p}}(t)=y_{\mathrm{p}}(t+\tau)$ is $\tau$ periodic.

\section{Forced vibration, ODE}

The forced stationary periodic motion $y_{\mathrm{p}}(t)$ can be calculated from the particular solution of Eq. (1) in case of stable machining ( $\left.\lim _{t \rightarrow \infty} \eta(t)=0\right)$ as the solution of the following ordinary differential equation (ODE)

$$
m \ddot{y}_{\mathrm{p}}(t)+c \dot{y}_{\mathrm{p}}(t)+k y_{\mathrm{p}}(t)=G_{\mathrm{p}}(t) f_{\mathrm{z}},
$$

where $G_{\mathrm{p}}(t)=G_{\mathrm{p}}(t+\tau)$ is a $\tau$-periodic directional force coefficient and $f_{\mathrm{Z}}$ is the feed per tooth [20].

\section{Variational system, DDE}

The stability of this stationary periodic motion $y_{\mathrm{p}}(t)$ can be analysed through the variational delay differential equation of Eq. (1) in the form

$$
m \ddot{\eta}(t)+c \dot{\eta}(t)+k \eta(t)=G(t)(\eta(t)-\eta(t-\tau)),
$$

which is a linear time-periodic delay differential equation (DDE). According to the Floquet theory of DDEs [8], the stability is determined by the corresponding monodromy operator $\mathscr{U}(\tau)$.

The state form of the general coordinate (see Eq. (2)) reads $\mathbf{y}(t)=\mathbf{y}_{\mathrm{p}}(t)+\boldsymbol{\eta}(t)$, where $\mathbf{y}(t)=[y(t) \dot{y}(t)]^{\mathrm{T}}, \mathbf{y}_{\mathrm{p}}(t)=$ $\left[y_{\mathrm{p}}(t) \dot{y}_{\mathrm{p}}(t)\right]^{\mathrm{T}}$ and $\boldsymbol{\eta}(t)=[\eta(t) \dot{\eta}(t)]^{\mathrm{T}}$. Introduce the discretized state vector of the perturbation $\boldsymbol{\eta}_{i}^{n} \in \mathbb{R}^{2 n \times 1}$ along a period $\tau$ as

$$
\boldsymbol{\eta}_{i}^{n}=\operatorname{col}_{j=1}^{n} \boldsymbol{\eta}\left(t_{i}-(j-1) \Delta t\right)
$$

where the number of sampled points is $n$, the discretized time step is $\Delta t=\tau / n$ and the time after $i^{\text {th }}$ period $t_{i}=t+i \tau$. Then the monodromy operator can be approximated by the monodromy matrix $\boldsymbol{\Phi}_{n} \in \mathbb{R}^{2 n \times 2 n}$ based on several numerical methods [8-10]. The connection of two subsequent discretized states can be given as a monodromy mapping in the form

$$
\boldsymbol{\eta}_{i+1}^{n}=\boldsymbol{\Phi}_{n} \boldsymbol{\eta}_{i}^{n}
$$

Eq. (4) is stable if and only if all characteristic multipliers $\mu_{n}$ (eigenvalues of $\boldsymbol{\Phi}_{n}$ ) have modulus less then 1 . 


\section{DETERMINATION OF THE DOMINANT MULTIPLIERS}

Our goal is to determine the monodromy matrix and the characteristic multipliers. Therefore, to capture the behaviour of the machining operation, the resulted vibration $y(t)$ is sampled with frequency $f_{\text {st }}$, as shown with blue curve in Fig 2 a.

\section{Stroboscopic section}

The sampling frequency is tuned to the period $\tau$ as $f_{\text {st }}=1 / \tau$. These stroboscopic sections are denoted by (see red dots in Fig. 2a)

$$
\mathbf{y}_{i}:=\mathbf{y}(i \tau), i \in \mathbb{N}
$$

According to Eq. (2), the sampled perturbation and stationary periodic motion are denoted by $\boldsymbol{\eta}_{i}:=\boldsymbol{\eta}(i \tau)$ and $\mathbf{y}_{\mathrm{p}, \mathrm{i}}:=\mathbf{y}_{\mathrm{p}}(i \tau)$, respectively. Due to the periodicity of the periodic term $\mathbf{y}_{\mathrm{p}}(t)=$ $\mathbf{y}_{\mathrm{p}}(t+\tau)$, thus, the sampled periodic terms are identical $\mathbf{y}_{\mathrm{p}, \mathrm{i}} \equiv \mathbf{y}_{\mathrm{p}}$. Therefore, Eq. (2) can be written in state form after stroboscopic mapping as

$$
\mathbf{y}_{i}=\mathbf{y}_{\mathrm{p}}+\boldsymbol{\eta}_{i}
$$

The discretization is based on the a continous system (see Eq. (4)), thus, $\boldsymbol{\Phi}$ is the projection of the monodromy operator, which approximates the subspace of dominant modes. Its eigenvalues estimate the dominant characteristic multipliers of the inifinite dimensional monodromy operator $\mathscr{U}(\tau)$. Note, that the sampling strategy is equivalent to the discretized state representation for $n=1$ (see Eq. (5)). In this case, the size of the monodromy matrix $\boldsymbol{\Phi}$ would be $2 \times 2$ with 2 dominant characteristic multipliers $\mu$, and the monodromy mapping is defined as

$$
\boldsymbol{\eta}_{i+1}=\boldsymbol{\Phi} \boldsymbol{\eta}_{i}
$$

\section{Monodromy mapping}

Note, that our goal is to determine the monodromy matrix directly from the measured signal. For that the monodromy matrix has to be expressed from Eq. (9). The basic concept is to extend the monodromy mapping with further elements [15]: a subsequent sampled perturbation state vector $\boldsymbol{\eta}_{i+2}$ as

$$
\left[\boldsymbol{\eta}_{i+1} \boldsymbol{\eta}_{\mathrm{i}+2}\right]=\boldsymbol{\Phi}\left[\boldsymbol{\eta}_{i} \boldsymbol{\eta}_{i+1}\right]
$$

then, the monodromy matrix can be determined by means of an inverse matrix calculation, reads as

$$
\boldsymbol{\Phi}=\left[\begin{array}{ll}
\boldsymbol{\eta}_{i+1} & \boldsymbol{\eta}_{\mathrm{i}+2}
\end{array}\right]\left[\boldsymbol{\eta}_{i} \boldsymbol{\eta}_{i+1}\right]^{-1}
$$
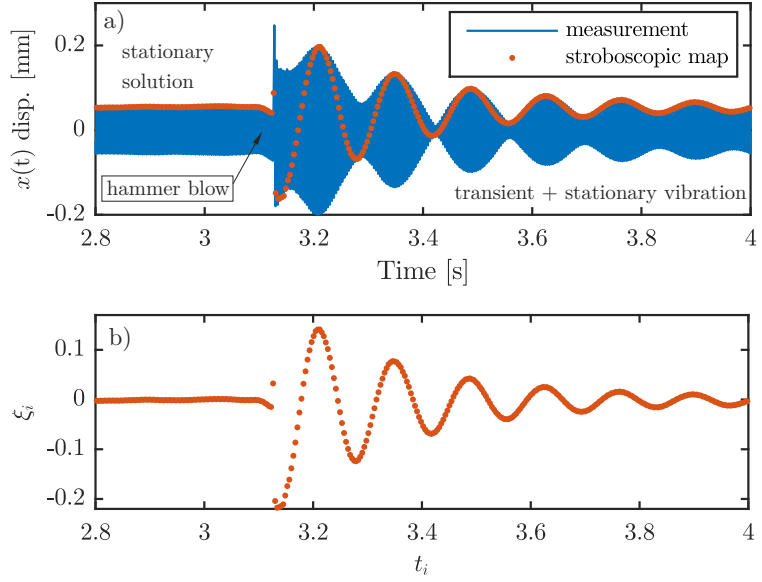

FIGURE 2. MEASUREMENT AND STROBOSCOPIC SECTION BEFORE AND AFTER HAMMER EXCITATION

In case of prefect measurement data, it would provide the necessary information, however, in practice some kind of averaging method is necessary in case of noisy data. In order to improve the determination of $\boldsymbol{\Phi}$, more then 2 sampled vector are used

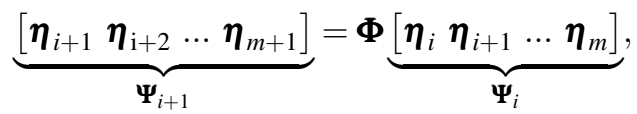

where $\boldsymbol{\Psi}_{i}$ and $\boldsymbol{\Phi}$ are $2 \times m$ and $2 \times 2$ matrices, respectively. Then $\boldsymbol{\Phi}$ can be expressed by the following formula

$$
\boldsymbol{\Phi}=\boldsymbol{\Psi}_{i+1} \boldsymbol{\Psi}_{i}^{\dagger}
$$

where $\boldsymbol{\Psi}_{i}^{\dagger}=\boldsymbol{\Psi}_{i}^{\mathrm{T}}\left(\boldsymbol{\Psi}_{i} \boldsymbol{\Psi}_{i}^{\mathrm{T}}\right)^{-1}$ is the generalized inverse (or MoorePenrose pseudoinverse) of $\boldsymbol{\Psi}_{i}$, which provides the solution in linear least square sense. Note, that $\Psi_{i}^{\dagger}$ is an $m \times 2$ matrix.

Theoretically, it is possible to perform the calculation for larger sampled points $(n>1)$ within one period and predict $2 n$ number of multipliers, if $m>2 n$. However, for large $n$, the convergence of the multipliers is insufficient because the corresponding transient motions die out quickly, hence, almost no information of these modes can be extracted from noisy measurement signal. However, only the dominant one is necessary for stability prediction and $n=1$ can be sufficient.

The problem is that the monodromy mapping (see Eq. (9)) is valid for the perturbation components $\boldsymbol{\eta}_{i}$ only, however, it cannot be measured directly from a milling operation. Therefore, it is required to separate the measured vibration $\mathbf{y}_{i}$ into periodic $\mathbf{y}_{\mathrm{p}}$ and perturbed components $\boldsymbol{\eta}_{i}$. 


\section{Determination of the periodic component}

In this section, the periodic term is derived in order to obtain the perturbed components, only. The perturbed term is given as (see Eq. (8))

$$
\boldsymbol{\eta}_{i}=\mathbf{y}_{i}-\mathbf{y}_{\mathrm{p}}
$$

and substituted into Eq. (9):

$$
\mathbf{y}_{i+1}-\mathbf{y}_{\mathrm{p}}=\boldsymbol{\Phi}\left(\mathbf{y}_{i}-\mathbf{y}_{\mathrm{p}}\right)
$$

from which, the periodic term can be expressed as

$$
\mathbf{x}_{\mathrm{p}}=(\mathbf{I}-\boldsymbol{\Phi})^{-1}\left(\mathbf{y}_{i+1}-\boldsymbol{\Phi} \mathbf{y}_{i}\right)
$$

However, $\boldsymbol{\Phi}$ is still unknown and the periodic term $\mathbf{y}_{\mathrm{p}}$ cannot be separated from $\mathbf{y}_{i}$ in this way.

From further investigation of (15), it can be seen that this mapping can be interpretable as a transformation (or mapping) which is composed of translation, rotation and scaling (stretching). The translation comes from the subtraction of the periodic term $\mathbf{y}_{\mathrm{p}}$, and the rotation and scaling (stretching) relate to the monodromy mapping itself.

\section{HOMOGENEOUS COORDINATE TRANSFORMATION}

Using so-called homogeneous coordinates and applying homogeneous coordinate transformation is an appropriate method for this kind of problems, since, a significant and practical feature of the homogeneous coordinate system is its fusion of the translation, rotation and scaling (stretching) of geometric objects $[21,22]$. Therefore, we extended the monodromy mapping in the following form

$$
\underbrace{\left[\begin{array}{c}
\mathbf{y}_{i+1} \\
1
\end{array}\right]}_{\mathbf{x}_{i+1}}=\underbrace{\left[\begin{array}{cc}
\boldsymbol{\Phi} & \mathbf{t}_{\mathrm{p}} \\
\mathbf{0}^{\mathrm{T}} & 1
\end{array}\right]}_{\hat{\boldsymbol{\Phi}}} \underbrace{\left[\begin{array}{c}
\mathbf{y}_{i} \\
1
\end{array}\right]}_{\mathbf{x}_{i}}
$$

where $\mathbf{x}_{i} \in \mathbb{R}^{3 \times 1}$ and $\hat{\boldsymbol{\Phi}} \in \mathbb{R}^{3 \times 3}$ are the homogeneous representation of state variable $\mathbf{y}_{i}$ and monodromy matrix $\boldsymbol{\Phi}$, respectively, and $\mathbf{0}$ is $2 \times 1$ column vector of zeros. The homogeneous coordinate transformation matrix $\hat{\boldsymbol{\Phi}}$ includes the monodromy matrix $\boldsymbol{\Phi}$ at the upper-left corner, and a vector quantity $\mathbf{t}_{\mathrm{p}} \in \mathbb{R}^{2 \times 1}$ associated with translational offset due to the subtraction of the periodic term at the upper-right corner. In this case, the transformation matrix $\hat{\boldsymbol{\Phi}}$ maps the periodic term into itself and the perturbed term according to the monodromy mapping. Note, that $\hat{\boldsymbol{\Phi}}$ always has one eigenvalue with real part equals to 1 due to the mapping of the periodic term.

Similarly as in the previous section, with more then 2 homogeneous coordinates are used in the form

$$
\underbrace{\left[\begin{array}{llll}
\mathbf{x}_{i+1} & \mathbf{x}_{\mathrm{i}+2} & \ldots & \mathbf{x}_{\mathrm{m}}
\end{array}\right]}_{\mathbf{X}_{i+1}}=\hat{\boldsymbol{\Phi}} \underbrace{\left[\begin{array}{llll}
\mathbf{x}_{i} & \mathbf{x}_{i+1} & \ldots & \mathbf{x}_{\mathrm{m}-1}
\end{array}\right]}_{\mathbf{X}_{i}},
$$

where $\mathbf{X}_{i}$ is a $3 \times m$ matrix. Then $\hat{\boldsymbol{\Phi}}$ can be expressed by

$$
\hat{\boldsymbol{\Phi}}=\mathbf{X}_{i+1} \mathbf{X}_{i}^{\dagger}
$$

Note, that after pseudoinverse calculation, $\hat{\boldsymbol{\Phi}}$ preserves its original form in the last row as $\left[\mathbf{0}^{\mathrm{T}} 1\right]$. Therefore, the monodromy matrix $\boldsymbol{\Phi}$ can be given by a partition of $\hat{\boldsymbol{\Phi}}$ according to Eq. (17). In addition, the periodic term $\mathbf{y}_{\mathrm{p}}$ can be calculated as

$$
\mathbf{y}_{\mathrm{p}}=(\mathbf{I}-\boldsymbol{\Phi})^{-1} \mathbf{t}_{\mathrm{p}}
$$

where $\mathbf{t}_{\mathrm{p}}=\mathbf{y}_{i+1}-\boldsymbol{\Phi} \mathbf{y}_{i}$. Note, that the derivation of the periodic term and the proof of Eq. (17) are presented in Appendix.

\section{EXPERIMENTAL STUDY}

In summary, with the help of the homogeneous coordinate transformation, the monodromy matrix and the corresponding characteristic multipliers can be approximated directly from measurement signal without applying any separation or subtraction of the periodic term. In this section, the proposed method is applied on a measurement of a stable machining process for a measurement setup which can be found in [19].

\section{Measurement problem}

Technological parameters of the applied experimental process was the followings: two-fluted tool $N=2$ with diameter $D=16 \mathrm{~mm}$ and straight edge during down-milling operation, radial immersion $a_{\mathrm{e}}=2 \mathrm{~mm}$, axial immersion $a_{\mathrm{p}}=1.5 \mathrm{~mm}$, feed per tooth $f_{\mathrm{z}}=0.05 \mathrm{~mm}$ and spindle speed $\Omega=8052 \mathrm{rpm}$.

During the milling process with straight tool path (see Fig. 1), the acceleration of the SDoF flexure is acquired by NI cDAQ9178 Chassis with NI 9234 Module at $52 \mathrm{kHz}$ sampling rate and PCB 352C23 type sensor. In the signal, double integration and high-pass filter is applied to obtain the position and velocity signal. Fitted modal parameters of the SDoF system are: natural frequency $\omega_{\mathrm{n}}=259.96 \mathrm{~Hz}$, modal mass $m=2.701 \mathrm{~kg}$ and relative damping ratio $\zeta=0.71 \%$.

For the proposed method, it is necessary to excite the system during stable machining operation. This is carried out by means 

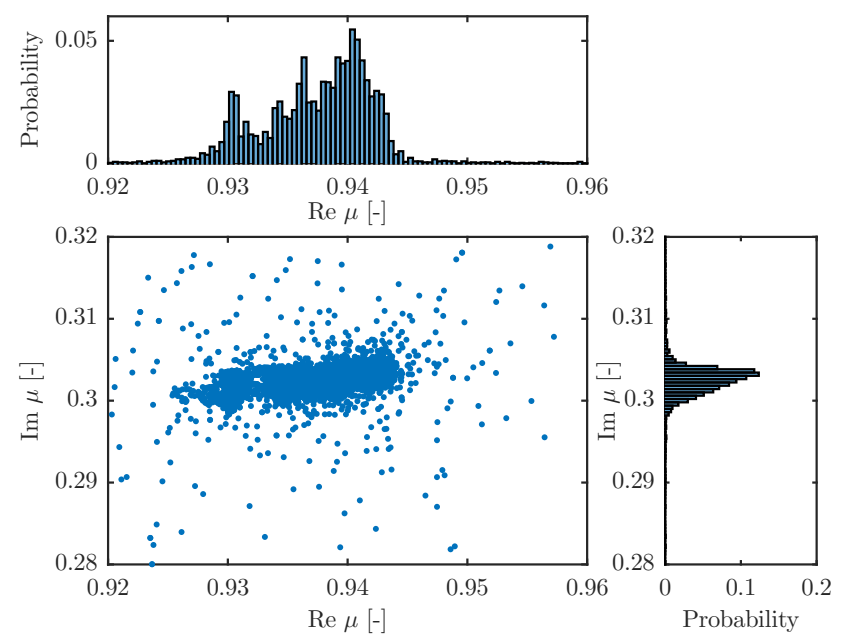

FIGURE 3. 5 THOUSANDS CALCULATED MULTIPLIER IN THE COMPLEX PLANE AND THEIR PROBABILITY DENSITY FUNCTION

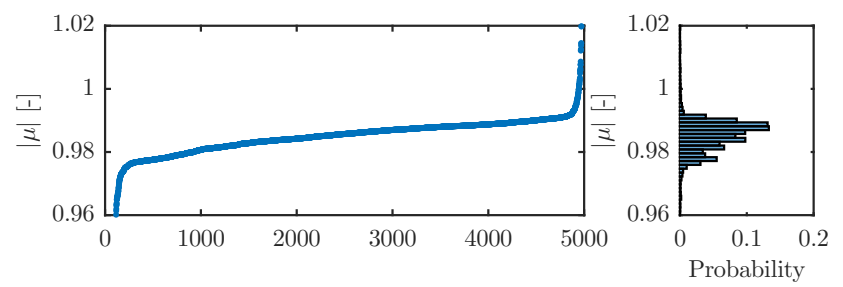

FIGURE 4. DETECTED MULTIPLIERS SORTED BY ITS MODULUS AND THE CORRESPONDING PROBABILITY DENSITY FUNCTION

of simple hammer blow on the text rig along the flexible direction $y$, and the resultant time signal is shown in Fig. 2a. The response after excitation is characterized by means of stroboscopic sampling with sampling frequency $f_{\text {str }}=268.4054 \mathrm{~Hz}$ (see Fig. 2a red dots), and the monodromy matrix can be calculated by Eq. (19).

The selected range of the sampled points can influence the monodromy matrix calculation because all measurements are loaded with uncertainty and inaccuracy. In order to investigate the accuracy of the method and to eliminate the influence of the human factor in the range selection, the dominant multipliers are determined for 5 thousand different cases. The selected range is generated by randomly varying initial point $\left(t_{i}\right)$ and random number of sampled data $m$.

Figure 3 presents the identified multipliers in the complex plane with their probability density function (PDF). It can be seen that the variance of PDF along the imaginary axis are smaller compared to the real one. Since, in the presented case, the imaginary part of the multiplier is more related to the fre-
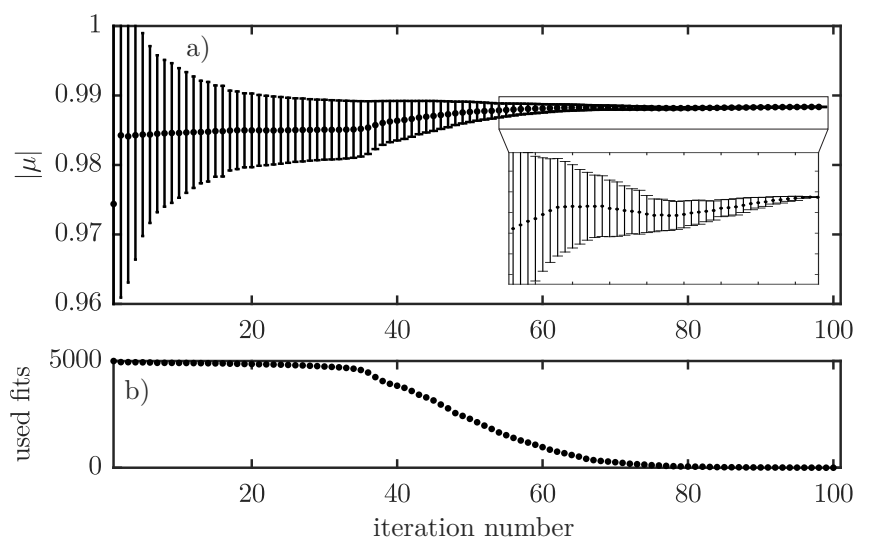

FIGURE 5. ITERATIVE METHOD TO APPROXIMATE THE DOMINANT MONODROMY MULTIPLIER

quency of the self excited vibration, it means that the method can give a better estimation for the chatter frequency, than for the decay ratio $|\mu|$. The deviation of modulus is visualized by plotting them in a sorted order, as shown in Fig. 4, which clearly shows, that most of the fitted values are dense in the range [0.975-0.99]. The reason of the large deviation in the fitted values are detailed in Subsection .

The actual value of the detected $\mu$ could be approximated by simple average of the 5 thousand fitted values, however, the mean value can be influenced significantly by badly fitted points which are relatively far from the average value. Therefore, those outlying points are dropped out, for which the distance from the mean value are larger then $95 \%$ of the maximal distance. The procedure is performed iteratively to reach better approximation and the result is visualized in Fig. 5a, where the absolute value and their standard deviation of the multiplier is plotted against the number of iteration. Figure $5 \mathrm{~b}$ shows the number of the remaining samples after dropping out the farthest ones.

\section{Accuracy and nonlinearities}

The reason for the high deviation in the detected multipliers can be found in the number of the selected points. The uncertainty is high if too short range is used for the monodromy matrix determination, therefore a few points are utilized for the calculation. This phenomena can be is noticed in Fig. 6, where the sampled time signal plotted in the bottom left panel, the used ranges are presented with vertical black lines in the bottom right panel and the corresponding detected multiplier visualized directly above it. One test case is highlighted with red color, where the selected range for the monodromy calculation is $i=[105-178]$ and the identified multiplier is $|\mu|=0.985$. Note, that the detected multipliers are sorted by the length of the used range (or the number of selected points $m$ ). This way, it can be noticed that for too small selected range, the deviation is signifi- 


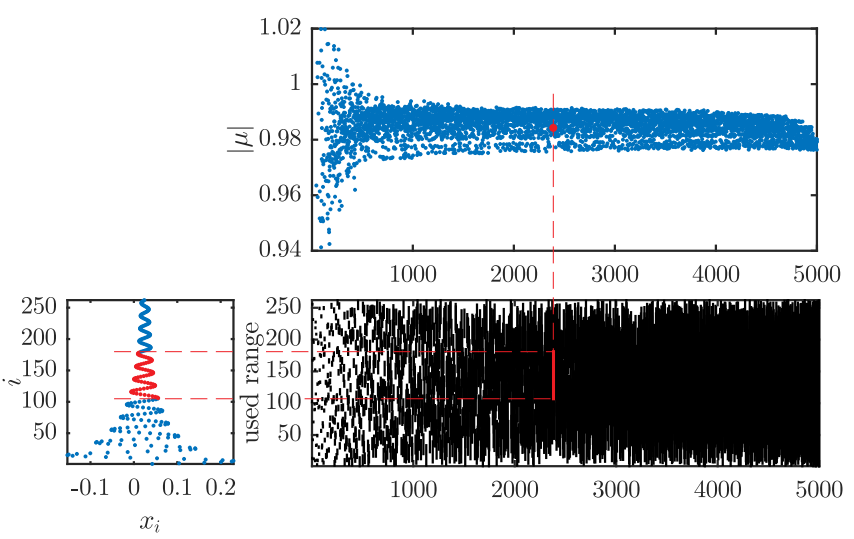

FIGURE 6. THE CALCULATED MULTIPLIERS ARE SORTED BY THE LENGTH OF THE SELECTED RANGE. A TEST CASE IS HIGHLIGHTED WITH RED COLOR TO PRESENT THE RANGE SELECTION PROCEDURE

cant. There is a critical range size $(m \approx 20)$, after which the deviation is almost constant. For further investigation, calculations based on range smaller then the critical one, the corresponding multipliers are neglected.

The source of this constant deviation can be revealed in Fig. 7, where the identified multipliers are sorted by the mid point of the selected range. Black color refers to all cases and red color considers cases only, where the length of the selected range is limited by the third of the maximum. This case is similar to a moving rectangular window function along the transient vibration. It can be seen from the calculated multipliers, that they are shifted from 0.975 to 0.99 as the window function moving from large amplitude vibrations to small amplitude ones. It means that the relating damping factor of the corresponding transient vibration getting lower in case of smaller vibration amplitude. One explanation can be that the magnitude of the multiplier (and also the corresponding damping ratio) depends on the amplitude of the perturbed vibration, which refers to the presence of nonlinearity.

\section{CONCLUSION}

In the present study, a chatter detection method is proposed, which is capable to give a quantitative measure of stability through the approximated dominant characteristic multiplier. Therefore, it is thoroughly comparable to the theoretically predicted stability charts. The projection of the monodromy operator, which approximates the subspace of dominant modes is introduced and the dominant multipliers are determined based on measured data with pseudoinverse calculation. Homogeneous coordinate representation is introduced for the parameter identification, which is suitable to separate directly the periodic and perturbed vibration in milling operations.
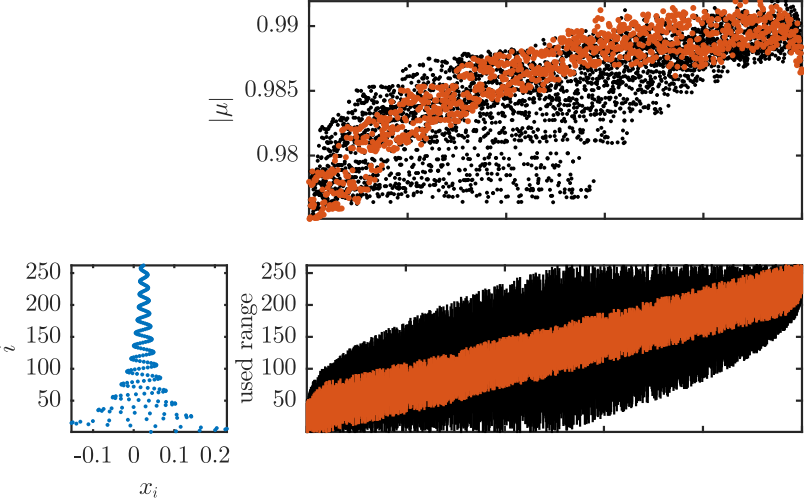

FIGURE 7. THE IDENTIFIED MULTIPLIERS ARE SORTED BY THE MID POINT OF THE SELECTED RANGE. RED COLOR REPRESENTS A MOVING WINDOW FUNCTION ALONG THE TRANSIENT VIBRATION AND THE CORRESPONDING DETECTED MULTIPLIERS

The accuracy of the calculation process is tested on a measurement case study, and the characteristic properties of the method is also explained in details. Finally, with the help of the provided method, vibration-amplitude-dependent nonlinear behavior of the perturbed solution is discovered.

\section{ACKNOWLEDGMENT}

This paper was supported by the Hungarian Scientific Research Fund - OTKA PD-112983 and the Janos Bolyai Research Scholarship of the Hungarian Academy of Sciences. The research leading to these results has received funding from the European Research Council under the European Unions Seventh Framework Programme (FP/2007-2013) / ERC Advanced Grant Agreement n. 340889.

\section{REFERENCES}

[1] Tobias, S., 1965. Machine-tool Vibration. Blackie, Glasgow.

[2] Tlusty, J., and Spacek, L., 1954. Self-excited vibrations on machine tools. Nakl. CSAV, Prague. in Czech.

[3] Stepan, G., 1989. Retarded dynamical systems: stability and characteristic functions. Longman Scientific and Technical.

[4] Mann, B. P., Young, K. A., Schmitz, T. L., and Dilley, D. N., 2004. "Simultaneous stability and surface location error predictions in milling". Journal of Manufacturing Science and Engineering, 127(3), pp. 446-453.

[5] Altintas, Y., and Budak, E., 1995. "Analytical prediction of stability lobes in milling". CIRP Annals - Manufacturing Technology, 44, pp. 357-362. 
[6] Budak, E., and Altintas, Y., 1998. "Analytical prediction of chatter stability in milling, part i: General formulation". Journal of Dynamic Systems Measurement and Control ASME, 120, pp. 22-30.

[7] Bachrathy, D., and Stepan, G., 2013. "Improved prediction of stability lobes with extended multi frequency solution". CIRP Annals - Manufacturing Technology, 62, pp. 411414.

[8] Insperger, T., and Stepan, G., 2011. Semi-discretization for time-delay systems, Vol. 178. Springer, New York.

[9] Butcher, E. A., Bobrenkov, O. A., Bueler, E., and Nindujarla, P., 2009. "Analysis of milling stability by the chebyshev collocation method: algorithm and optimal stable immersion levels". Journal of Computational and Nonlinear Dynamics - ASME, 4, p. 031003.

[10] Khasawneh, F. A., and Mann, B. P., 2011. "A spectral element approach for the stability of delay systems". International Journal for Numerical Methods in Engineering, 87, p. 566952.

[11] Quintana, G., and J, J. C., 2011. "Chatter in machining processes: A review". International Journal of Machine Tools and Manufacture, 51(5), p. 363376.

[12] Altintas, Y., and Chan, P. K., 1992. "In-process detection and suppression of chatter in milling". International Journal of Machine Tools and Manufacture, 32(3), pp. 329347.

[13] Delio, T., Tlusty, J., and Smith, S., 1992. "Use of audio signals for chatter detection and control". Journal of Engineering for Industry, 114(2), pp. 146-157.

[14] Bediaga, I., Munoa, J., Hernandez, J., and de Lacalle, L. L., 2009. "Spindle speed selection strategy to obtain stability in high-speed milling". International Journal of Machine Tools and Manufacture, 49(5), pp. 384-394.

[15] Mann, B. P., and Young, K. A., 2006. "An empirical approach for delayed oscillator stability and parametric identification". Proceedings of the Royal Society of London A: Mathematical, Physical and Engineering Sciences, 462(2071), pp. 2145-2160.

[16] Schmitz, T. L., 2003. "Chatter recognition by a statistical evaluation of the synchronously sampled audio signal". Journal of Sound and Vibration, 262, p. 721730.

[17] Honeycutt, A., and Schmitz, T. L., 2016. "A new metric for automated stability identification in time domain milling simulation". Journal of Manufacturing Science and Engineering, 138(7), p. 074501.

[18] Khalifa, O. O., Densibali, A., and Faris, W., 2006. "Image processing for chatter identification in machining processes". The International Journal of Advanced Manufacturing Technology, 31, p. 443449.

[19] Bayly, P. V., Mann, B. P., Schmitz, T. L., Peters, D., Stepan, G., and Insperger, T., 2002. "Effects of radial immersion and cutting direction on chatter instability in end-milling".
Proceedings of the 2002 ASME International Mechanical Engineering Congress and Exposition(IMECE200234116).

[20] Altintas, Y., 2012. Manufacturing Automation; Metal Cutting Mechanics, Machine Tool Vibrations, and CNC Design, 2nd Edition. Cambridge University Press.

[21] Veblen, O., and , J. Y., 1956. A Mathematical Science. In The World of Mathematics, Vol. 3. Simon and Schuster, New York.

[22] Aleksandrov, A. D., Kolmogorov, A. N., and Lavientev, M. A., 1969. Mathematics, its Content, Methods and Meaning. MIT Press.

\section{APPENDIX: Determination of the periodic component}

The monodromy mapping using homogeneous coordinates (see Eq. (17)) can be given as

$$
\underbrace{\left[\begin{array}{c}
\boldsymbol{\eta}_{i+1} \\
1
\end{array}\right]}_{\boldsymbol{\xi}_{i+1}}+\underbrace{\left[\begin{array}{c}
\mathbf{y}_{\mathrm{p}} \\
0
\end{array}\right]}_{\mathbf{x}_{\mathrm{p}}}=\hat{\boldsymbol{\Phi}}(\underbrace{\left[\begin{array}{c}
\boldsymbol{\eta}_{i} \\
1
\end{array}\right]}_{\boldsymbol{\xi}_{i}}+\underbrace{\left[\begin{array}{c}
\mathbf{y}_{\mathrm{p}} \\
0
\end{array}\right]}_{\mathbf{x}_{\mathrm{p}}}) .
$$

The homogeneous coordinate transformation matrix (or mapping) $\hat{\boldsymbol{\Phi}}$ can be decomposed as

$$
\hat{\boldsymbol{\Phi}}=\hat{\boldsymbol{\Phi}}_{\xi}+\hat{\boldsymbol{\Phi}}_{\mathrm{p}}
$$

where $\hat{\boldsymbol{\Phi}}_{\xi}$ relates to the monodromy mapping between the perturbed terms, formulated as

$$
\hat{\boldsymbol{\Phi}}_{\xi}=\left[\begin{array}{ll}
\boldsymbol{\Phi} & 0 \\
\mathbf{0}^{\mathrm{T}} & 1
\end{array}\right],
$$

and $\hat{\boldsymbol{\Phi}}_{\mathrm{p}}$ connects to the translation (subtraction) of the periodic term, reads as

$$
\hat{\boldsymbol{\Phi}}_{\mathrm{p}}=\left[\begin{array}{cc}
\mathbf{O} & \mathbf{t}_{\mathrm{p}} \\
\mathbf{0}^{\mathrm{T}} & 0
\end{array}\right]
$$

where $\mathbf{O}$ is an $2 \times 2$ null matrix. Substitute Eq. (23) and (24) into Eq. (21) yields

$$
\boldsymbol{\xi}_{i+1}+\mathbf{x}_{\mathrm{p}}=\hat{\boldsymbol{\Phi}}_{\xi} \boldsymbol{\xi}_{i}+\hat{\boldsymbol{\Phi}}_{\xi} \mathbf{x}_{\mathrm{p}}+\hat{\boldsymbol{\Phi}}_{\mathrm{p}} \boldsymbol{\xi}_{i}+\hat{\boldsymbol{\Phi}}_{\mathrm{p}} \mathbf{x}_{\mathrm{p}}
$$

Using, that $\hat{\boldsymbol{\Phi}}_{\xi} \boldsymbol{\xi}_{i}=\boldsymbol{\xi}_{i+1}, \hat{\boldsymbol{\Phi}}_{\mathrm{p}} \boldsymbol{\xi}_{i}=\left[\mathbf{t}_{\mathrm{p}} 0\right]^{\mathrm{T}}$ and $\hat{\boldsymbol{\Phi}}_{\mathrm{p}} \mathbf{x}_{\mathrm{p}}=\mathbf{0}$, the periodic term $\mathbf{x}_{\mathrm{p}}$ can be expressed as

$$
\mathbf{x}_{\mathrm{p}}=\left(\mathbf{I}-\hat{\boldsymbol{\Phi}}_{\xi}\right)^{-1}\left[\begin{array}{c}
\mathbf{t}_{\mathrm{p}} \\
0
\end{array}\right]
$$


Further investigation of Eq. (26), it is straightforward that the periodic component can be calculated as.

$$
\mathbf{y}_{\mathrm{p}}=(\mathbf{I}-\boldsymbol{\Phi})^{-1} \mathbf{t}_{\mathrm{p}}
$$

Using Eq. (16) and Eq. (27), $\mathbf{t}_{\mathrm{p}}$ can be expressed as

$$
\mathbf{t}_{\mathrm{p}}=\mathbf{y}_{i+1}-\boldsymbol{\Phi} \mathbf{y}_{i}
$$

